\title{
ON THE CUBOID PROBLEM, THE FINAL CHAPTER
}

\author{
Alex Nguhi ${ }^{1} 1$
}

\begin{abstract}
This paper builds on a previous finding regarding the cuboid problem. It was postulated that the cuboid problem is a divisibility by 3 problem. The idea is expanded fully to display in detail the difficulty of such a cuboid existing even under the most extreme circumstances. The cuboid problem is a paradox and it shouldn't exist at all.
\end{abstract}

\section{INTRODUCTION}

This paper assumes an elementary understanding of the cuboid problem since it is somewhat a continuation of a previous paper [1]. The focus will be on the Diophantine analysis of the equation $a^{2}+b^{2}+e^{2}=I^{2}$. The Cuboid Problem is related to Euler Bricks, [2,3].

\section{SQUARES REVISITED.}

\section{Lemma 2.1}

For any arbitrary integer $n$ for $n \in \mathbf{N}$ with $n>1$ and not divisible by 3, then $n^{2} \bmod 3=1$

\section{Proof:}

Any $n$ has modulus of 0,1 or 2 when divided by 3 . For mod 0 , lemma 2.1 is trivial because a number divisible by 3 also has a square divisible by 3 or by 9 for that matter.

For modulus 1, when $n$ is squared it, the modulus is preserved. This can be proven using a number $p$ such that $n=k p+1$ where $\mathrm{p}$ is divisible by 3 and $k$ is just a scalar which may or may not be divisible by 3 .

Therefore

$$
n^{2}=(k p+1)^{2}=k^{2} p^{2}+2 k p+1
$$

From the above equation is clear that the modulus is preserved because $k p$ and $2 k p$ are divisible by 3 .

For Modulus 2,

$$
n^{2}=(k p+2)^{2}=k^{2} p^{2}+4 k p+4
$$

alexnguhi@gmail.com 
Like the previous case $k p$ and $4 k p$ are divisible by 3 , but the last constant is 4 which is of modulus 1 . Hence, the lemma is true.

\section{Proposition 2.1} 3

For any Pythagorean triples, $a<b<c$, at least one element is divisible by

In the last paper this was concretely proven using other methods and postulated that divisibility by 3 is reserved only for slots $a, b$

Using lemma 2.1 we can continue the exploration. We'll also have to remember that archetypal equations relating to certain radii determine the distribution of primitive Pythagorean triples.

Let's delve deeper. Consider a case where $a, b$ are of modulus 1 , this automatically means that the sum of their squares, $c^{2}$ will be of modulus 2 , thereby be in violation of the behavior of any squares because the lemma clearly says that modulus must be 1 .

This means the system needs a correction mechanism that helps preserve the modulus and that happens to be a number divisible by 3 . This is a sufficient proof of the proposition.

This goes in tandem with the next proposition.

\section{Proposition 2.2}

For any Pythagorean triples, at least one integer is divisible by 5.

In the first paper this was sufficiently proven for Archetype 1 using terminal digits, [1].

The modulus method is not sufficient for divisibility by 5 because of complications arising from the radii. Nonetheless we can make useful deductions.

Consider for a number $a$ where $a=5 k+z$ where $z=\operatorname{a} \bmod 5$ that is $\mathrm{z}=$ $0,1,2,3,4$. Like in the previous case, $k$ is just a scalar.

Squaring $a$

$a^{2}=(5 k+z)^{2}=25 k^{2}+10 k z+z^{2}$

We can conclude that the divisibility depends on $z$, therefore,

when :

$\mathrm{z}=0$, the solution is trivial

$\mathrm{z}=1, a^{2} \bmod 5=1$ since $z^{2}=1$

$\mathrm{z}=2, a^{2} \bmod 5=4$ since $z^{2}=4$

$\mathrm{z}=3, a^{2} \bmod 5=4$ since $z^{2}=9$

$\mathrm{z}=4, a^{2} \bmod 5=1$ since $z^{2}=16$ 
The major takeaway is that 1,4 and the trivial 0 are the only moduli for any square number when divided by 5 .

A combined mechanism for both divisibility by 3 and 5 can be developed.

Such a model might propose that if $a$ is divisible by 3 and 5 then $b^{2}$ must have a modulus of 1 when divided by 5 so that it preserves the moduli for the other side, $c^{2}$. This is because $a^{2}=c^{2}-b^{2}$ and $a^{2} \bmod 5=0$

$$
\begin{gathered}
a^{2}=c^{2}-b^{2} \\
0=1-1
\end{gathered}
$$

For instance the primitive triples $(11,60,61),(161,240,289)$ and $(60,91,109)$.

The same preservation principle happens when $a$ is only divisible by 5 and not 3 for instance $(20,99,101)$

\section{THE CUBOID PROBLEM}

The figure below shows a hypothetical cuboid from which the Diophantine equation $a^{2}+b^{2}+e^{2}=I^{2}$ is derived.

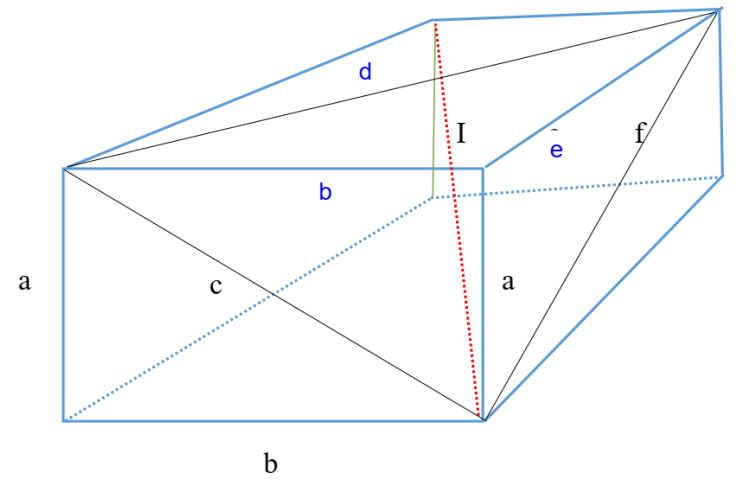

Figure 1: cuboid

Previously it was established in the cuboid problem that three triangle share the shortest side, [1]. The triangles are $(\mathrm{a}, \mathrm{b}, \mathrm{c}),(\mathrm{a}, \mathrm{e}, \mathrm{f})(\mathrm{a}, \mathrm{d}, \mathrm{I})$ with a fourth triangle formed by the sandwiched elements $(\mathrm{b}, \mathrm{e}, \mathrm{d})$.

By Pythagorean theorem ,

$$
a^{2}+d^{2}=I^{2}
$$


We can substitute $d$ because $b^{2}+e^{2}=d^{2}$

This gives $a^{2}+b^{2}+e^{2}=I^{2}$

\subsection{DIVISIBILITY BY 3 and THE CUBOID PROBLEM}

Divisibility by 3 alone can prove that the cuboid problem is perhaps a cuboid paradox that is inherently inconsistent.

\section{Proof of the Cuboid Paradox}

Consider a case where only $a$ is divisible by 3 , this means that the summation results in a modulus of 2 which is a violation of lemma 2.1 since no square number has a modulus of 2 .

$$
\begin{gathered}
a^{2}+b^{2}+e^{2}=I^{2} \\
\mathbf{0}+\mathbf{1}+\mathbf{1}=\mathbf{2}
\end{gathered}
$$

In the same triangle $(\mathrm{b}, \mathrm{e}, \mathrm{d})$

$$
\begin{gathered}
b^{2}+e^{2}=d^{2} \\
\mathbf{1}+\mathbf{1}=\mathbf{2}
\end{gathered}
$$

A second case can be proven by lemma 2.1 straight away when $a$ is not divisible by 3 , this too results in a paradox for $(b, e, d)$ because $(\mathrm{b}, \mathrm{e})$ must be divisible by 3 in triangles $(a, b, c)$ and $(a, e, f)$. It'd also require us to obtain an non-existent integer, $d^{2}$ which has a modulus of 0 , a number divisible by 3 . This gives a conundrum because we need $d^{2}$ to be of mod 1 .

$$
\begin{gathered}
b^{2}+e^{2}=d^{2} \\
0+0 \neq 1
\end{gathered}
$$

Still, are there any two numbers divisible by 3 whose squares add up to another square number divisible by 3 ?

$$
\begin{gathered}
b^{2}+e^{2}=d^{2} ? \\
\mathbf{0}+\mathbf{0}=\mathbf{0}
\end{gathered}
$$

Looking closely, this means that $3^{n}$ is just a scalar in triangle (b,e,d) and it can be scaled out completely before the squaring is done. The probability of such a combination existing is close to none because it leads to the same case scenarios discussed above. If not, a fine tuning would be done in such way that :

$$
\begin{gathered}
b^{2}+e^{2}=d^{2} \\
\left(k_{1} 3^{q}\right)^{2}+\left(k_{2} 3^{r}\right)^{2}=\left(k_{3} 3^{q}\right)^{2}
\end{gathered}
$$

For some constants $k_{i}$ and $q<r$ for $k_{i}, q, r \in \mathbf{N}$. 
This ensures that after complete factorization $e$ is still divisible by 3 to guarantee the preservation of the moduli. Such numbers might exist but not in the radii of Pythagorean triples. This means they'll affect the other dimensions $(\mathbf{a}, \mathbf{c}, \mathbf{f}, \mathbf{I})$

\section{CONCLUSION}

Due to divisibility by 3 obtaining a perfect cuboid results in a paradox.

\section{REFERENCES.}

1. Nguhi, Alex and Cleophas, Kweyu, On The Pythagorean Triples' Equations and the perfect cuboid problem. OSF Preprints . April 4 (2021)

2. Weisstein, E.W(2002).Cuboid.https://mathworld.wolfram.com

3. Butler, Bill. "Durango Bill's The 'Integer Brick' Problem(The Euler Brick Problem". https://www.durangobill.com/IntegerBrick.html 